\title{
Cosmological Constant and Universe
}

\section{El-Lakany MA*}

Physics Department, Faculty of Science, Cairo University, Egypt

*Corresponding author: Mohammed A El-Lakany, Physics Department, Faculty of Science, Cairo University, Giza, Egypt, Email: mohammed_ellakani@yahoo.com

\section{Mini Review}

Volume 4 Issue 2

Received Date: June 25, 2020

Published Date: August 11, 2020

\section{Abstract}

Cosmological constant is proportional to the sum of binding energy and its absolute value. If we supposed that the absolute value of binding energy is a function of $\left(\mathrm{A}, Z^{\prime}\right)$ instead of $(\mathrm{A}, \mathrm{Z})$ we have vacuum electric energy density $\rho_{1}$ as a function of $(\mathrm{A}$, $Z$ ) and Vacuum magnetic energy density $\rho_{2}$ as a function of $\left(\mathrm{A}, Z^{\prime}\right) \cdot \rho_{1}$ is proportional to the square value of electric field in empty space and $\rho_{2}$ is proportional to the square value of magnetic field. Using these definitions for $\rho_{1}$ and $\rho_{2}$ giving the exponential formula for the cosmological constant, but cosmological constant must be real value since $\rho_{1}$ and $\rho_{2}$ are real values and then it is a sine function.

Keywords: Unification of Gravity and Electromagnetism; Nuclear and particle physics; Astrophysics and cosmology

\section{Introduction}

Our universe is a huge collection of groups of nuclei. Cosmic rays are particles which have origin outside the Earth's atmosphere and they are nuclei of various kinds. Cosmic rays are outcome products of nuclear reactions and radioactive decay for gravitational objects [1-3]. Gravitational objects are nuclei having protons and neutrons numbers. Nucleus is a construction model for the universe. Elementary particles, nuclei, planets, stars, black holes, galaxies and clusters are the constituents of the universe. These objects constitute groups of objects and every group has a number of subgroups.

\section{Vacuum Electric Energy and Vacuum Magnetic Energy}

$\rho_{1}$ and $\rho_{2}$ are functions of the same atomic mass number (A) for the same nucleus and have the same atomic number in case of the ground state of the nucleus (atomic state) i.e. $\left(Z=Z_{0}=Z^{\prime}\right)$ but have different atomic numbers in case of excited state. Every ground state and their isobars compose a subgroup. This subgroup have nuclei of $Z=Z_{0}, Z_{1}, Z_{2}, Z_{3} \ldots \ldots, Z^{\prime}=Z_{0}, Z$ is the number of protons for vacuum electric energy and $Z^{\prime}$ is the atomic number for vacuum magnetic energy [4]. Cosmological constant equal to zero in case of the ground state but doesn't equal to zero for excited states and may be positive or negative value. Cosmological constant isn't one value as discussed by previous papers in this context but every nucleus has a specific value for it and varies from nucleus to another.

\section{Cosmological Constant}

The cosmological constant is given by

$$
\Lambda=\frac{8 \pi G}{c^{4}}\left(\frac{B \cdot E \cdot}{A}\right)+\frac{8 \pi G}{c^{4}}\left|\frac{B \cdot E .}{A}\right|
$$

If $Z, Z^{\prime}$ are the atomic numbers for vacuum electric energy and vacuum magnetic energy for the same nucleus, we find

$$
\begin{gathered}
\frac{B . E .}{A}=\left[\frac{M_{N}}{A}-\frac{Z}{A} m_{p}-\frac{(\mathrm{A}-\mathrm{Z})}{A} \mathrm{~m}_{n}\right] c^{2} \\
\left|\frac{B . E .}{A}\right|=\left[\frac{Z^{\prime}}{A} m_{p}+\frac{\left(\mathrm{A}-\mathrm{Z}^{\prime}\right)}{A} \mathrm{~m}_{n}-\frac{M_{N}}{A}\right] c^{2}
\end{gathered}
$$


And cosmological constant become

$$
\begin{gathered}
\Lambda=\frac{8 \pi G}{c}\left(\left[\frac{M_{N}}{\mathrm{~A}}-\frac{Z}{\mathrm{~A}} m_{p}-\mathrm{m}_{n}+\frac{\mathrm{Z}}{\mathrm{A}} \mathrm{m}_{n}\right] c^{2}+\left[\frac{Z^{\prime}}{\mathrm{A}} m_{p}+\mathrm{m}_{n}-\frac{\mathrm{Z}^{\prime}}{\mathrm{A}} \mathrm{m}_{n}-\frac{M_{N}}{\mathrm{~A}}\right] c^{2}\right) \\
\Lambda=\frac{8 \pi G}{c^{4}}\left(-\frac{Z}{\mathrm{~A}} m_{p}+\frac{\mathrm{Z}}{\mathrm{A}} \mathrm{m}_{n}+\frac{Z^{\prime}}{\mathrm{A}} m_{p}-\frac{\mathrm{Z}^{\prime}}{\mathrm{A}} \mathrm{m}_{n}\right) c^{2}(5) \\
\Lambda=\frac{8 \pi G}{c^{4}}\left(\frac{\mathrm{Z} c^{2}}{\mathrm{~A}}\left(\mathrm{~m}_{n}-m_{p}\right)-\frac{Z^{\prime} c^{2}}{\mathrm{~A}}\left(\mathrm{~m}_{n}-m_{p}\right)\right)(6) \\
\Lambda=\frac{8 \pi G}{c^{4}}\left(\rho_{1}+\rho_{2}\right)(7)
\end{gathered}
$$

Cosmological constant is a construction of electromagnetic field strength tensor and given by

$$
\begin{array}{r}
\Lambda=\frac{2 \pi G}{c} F^{\delta \lambda} F_{\delta \lambda}(8) \\
\Lambda=\frac{8 \pi G}{c^{4}}\left(-\frac{1}{2} E^{2}+\frac{1}{2} B^{2}\right)
\end{array}
$$

Density of vacuum electric energy is given by

$$
\rho_{1}=-\frac{1}{2} E^{2}(10)
$$

And density of vacuum magnetic energy can be written as

$$
\rho_{2}=\frac{1}{2} B^{2}
$$

Vacuum electric energy and vacuum magnetic energy have general coordinates $\left(x_{1}, x_{2}, x_{3}, x_{4}\right),\left(x_{1}^{\prime}, x_{2}^{\prime}, x_{3}^{\prime}, x_{4}^{\prime}\right)$ so that electric and magnetic fields in empty space are given by

$$
\begin{aligned}
& \vec{E}=\vec{E}_{01} e^{i\left(k_{1} x_{1}-\omega_{1} x_{4}\right)}+\vec{E}_{02} e^{i\left(k_{2} x_{2}-\omega_{2} x_{4}\right)}+\vec{E}_{03} e^{i\left(k_{3} x_{3}-\omega_{3} x_{4}\right)} \\
& \vec{B}=\vec{B}_{01} e^{i\left(k_{1} x_{1}^{\prime}-\omega_{1} x_{4}^{\prime}\right)}+\vec{B}_{02} e^{i\left(k_{2} x_{2}^{\prime}-\omega_{2} x_{4}^{\prime}\right)}+\vec{B}_{03} e^{i\left(k_{3} x_{3}^{\prime}-\omega_{3} x_{4}^{\prime}\right)}
\end{aligned}
$$

And their square values are given by

$$
\begin{aligned}
& E^{2}=e^{2 i\left(k_{1} x_{1}-\omega_{1} x_{4}\right)}+e^{2 i\left(k_{2} x_{2}-\omega_{2} x_{4}\right)}+e^{2 i\left(k_{3} x_{3}-\omega_{3} x_{4}\right)} \\
& B^{2}=e^{2 i\left(k_{1} x_{1}^{\prime}-\omega_{1} x_{4}^{\prime}\right)}+e^{2 i\left(k_{2} x_{2}^{\prime}-\omega_{2} x_{4}^{\prime}\right)}+e^{2 i\left(k_{3} x_{3}^{\prime}-\omega_{3} x_{4}^{\prime}\right)}
\end{aligned}
$$

Wave frequency and wave vector are given by

$$
\omega=\omega_{1}+\omega_{2}+\omega_{3}, \vec{k}=\left(k_{1}, k_{2}, k_{3}\right)
$$

The exponential formula for the cosmological constant can be written as

$$
\begin{aligned}
& \Lambda=\frac{8 \pi G}{c^{4}}\left[-\frac{1}{2} e^{2 i\left(k_{1} x_{1}-\omega_{1} x_{4}\right)}-\frac{1}{2} e^{2 i\left(k_{2} x_{2}-\omega_{2} x_{4}\right)}-\frac{1}{2} e^{2 i\left(k_{3} x_{3}-\omega_{3} x_{4}\right)}\right. \\
& \left.+\frac{1}{2} e^{2 i\left(k_{1} x_{1}^{\prime}-\omega_{1} x_{4}^{\prime}\right)}+\frac{1}{2} e^{2 i\left(k_{2} x_{2}^{\prime}-\omega_{2} x_{4}^{\prime}\right)}+\frac{1}{2} e^{2 i\left(k_{3} x_{3}^{\prime}-\omega_{3} x_{4}^{\prime}\right)}\right]
\end{aligned}
$$

Let's suppose that $\omega_{1}=\omega_{2}=\omega_{3}, k_{1}=k_{2}=k_{3}, x_{1}=x_{2}=x_{3}$ and $x_{1}^{\prime}=x_{2}^{\prime}=x_{3}^{\prime}$ we find

$$
\begin{aligned}
& \Lambda=\frac{8 \pi G}{c}\left(\frac{3}{2} e^{2 i\left(k_{1} x_{1}^{\prime}-\omega_{1} x_{4}^{\prime}\right)}-\frac{3}{2} e^{2 i\left(k_{1} x_{1}-\omega_{1} x_{4}\right)}\right) \\
& \Lambda=\frac{8 \pi G}{c^{4}}\left(\frac{3}{2}\right)\left(e^{2 i\left(k_{1} x_{1}^{\prime}-\omega_{1} x_{4}^{\prime}\right)}-e^{2 i\left(k_{1} x_{1}-\omega_{1} x_{4}\right)}\right)
\end{aligned}
$$

The exponential formula for the two complex numbers in the previous equation can be written as

$$
\begin{aligned}
& e^{2 i\left(k_{1} x_{1}^{\prime}-\omega_{1} x_{4}^{\prime}\right)}=\cos 2\left(k_{1} x_{1}^{\prime}-\omega_{1} x_{4}^{\prime}\right)+i \sin 2\left(k_{1} x_{1}^{\prime}-\omega_{1} x_{4}^{\prime}\right) \\
& e^{2 i\left(k_{1} x_{1}-\omega_{1} x_{4}\right)}=\cos 2\left(k_{1} x_{1}-\omega_{1} x_{4}\right)+i \sin 2\left(k_{1} x_{1}-\omega_{1} x_{4}\right)
\end{aligned}
$$

Cosmological constant in terms of sine and cosine functions are given by

$$
\begin{aligned}
& \Lambda=\frac{8 \pi G}{c}\left(\frac{3}{2}\right)\left[\cos 2\left(k_{1} x_{1}^{\prime}-\omega_{1} x_{4}^{\prime}\right)+i \sin 2\left(k_{1} x_{1}^{\prime}-\omega_{1} x_{4}^{\prime}\right)\right. \\
& \left.-\cos 2\left(k_{1} x_{1}-\omega_{1} x_{4}\right)-i \sin 2\left(k_{1} x_{1}-\omega_{1} x_{4}\right)\right] \\
& =\frac{8 \pi G}{c}\left(\frac{3}{2}\right)\left[\cos 2\left(k_{1} x_{1}^{\prime}-\omega_{1} x_{4}^{\prime}\right)-\cos 2\left(k_{1} x_{1}-\omega_{1} x_{4}\right)\right. \\
& \left.+i\left(\sin 2\left(k_{1} x_{1}^{\prime}-\omega_{1} x_{4}^{\prime}\right)-\sin 2\left(k_{1} x_{1}-\omega_{1} x_{4}\right)\right)\right] \\
& =\frac{8 \pi G}{c}\left(\frac{3}{2}\right)\left[-2 \sin \left(\left(k_{1} x_{1}^{\prime}-\omega_{1} x_{4}^{\prime}\right)+\left(k_{1} x_{1}-\omega_{1} x_{4}\right)\right) \sin \left(\left(k_{1} x_{1}^{\prime}-\omega_{1} x_{4}^{\prime}\right)-\left(k_{1} x_{1}-\omega_{1} x_{4}\right)\right)\right. \\
& \left.+2 i \cos \left(\left(k_{1} x_{1}^{\prime}-\omega_{1} x_{4}^{\prime}\right)+\left(k_{1} x_{1}-\omega_{1} x_{4}\right)\right) \sin \left(\left(k_{1} x_{1}^{\prime}-\omega_{1} x_{4}^{\prime}\right)-\left(k_{1} x_{1}-\omega_{1} x_{4}\right)\right)\right] \\
& =\frac{8 \pi G}{c}(-3)\left[\sin \left(\left(k_{1} x_{1}^{\prime}-\omega_{1} x_{4}^{\prime}\right)+\left(k_{1} x_{1}-\omega_{1} x_{4}\right)\right) \sin \left(\left(k_{1} x_{1}^{\prime}-\omega_{1} x_{4}^{\prime}\right)-\left(k_{1} x_{1}-\omega_{1} x_{4}\right)\right)\right. \\
& \left.-i \cos \left(\left(k_{1} x_{1}^{\prime}-\omega_{1} x_{4}^{\prime}\right)+\left(k_{1} x_{1}-\omega_{1} x_{4}\right)\right) \sin \left(\left(k_{1} x_{1}^{\prime}-\omega_{1} x_{4}^{\prime}\right)-\left(k_{1} x_{1}-\omega_{1} x_{4}\right)\right)\right] \\
& =-\frac{24 \pi G}{c} \sin \left(\left(k_{1} x_{1}^{\prime}-\omega_{1} x_{4}^{\prime}\right)-\left(k_{1} x_{1}-\omega_{1} x_{4}\right)\right)\left[\sin \left(\left(k_{1} x_{1}^{\prime}-\omega_{1} x_{4}^{\prime}\right)+\left(k_{1} x_{1}-\omega_{1} x_{4}\right)\right)\right. \\
& \left.-i \cos \left(\left(k_{1} x_{1}^{\prime}-\omega_{1} x_{4}^{\prime}\right)+\left(k_{1} x_{1}-\omega_{1} x_{4}\right)\right)\right]
\end{aligned}
$$

(21) [5] 
It is appeared from equation (6) that equation (21) must be real number not complex number i.e.

$\Lambda=-\frac{24 \pi G}{c^{4}} \sin \left(\left(k_{1} x_{1}^{\prime}-\omega_{1} x_{4}^{\prime}\right)-\left(k_{1} x_{1}-\omega_{1} x_{4}\right)\right) \sin \left(\left(k_{1} x_{1}^{\prime}-\omega_{1} x_{4}^{\prime}\right)+\left(k_{1} x_{1}-\omega_{1} x_{4}\right)\right)$

(22)

$$
\begin{gathered}
\cos \left(\left(k_{1} x_{1}^{\prime}-\omega_{1} x_{4}^{\prime}\right)+\left(k_{1} x_{1}-\omega_{1} x_{4}\right)\right)=0 \\
\left(\left(k_{1} x_{1}^{\prime}-\omega_{1} x_{4}^{\prime}\right)+\left(k_{1} x_{1}-\omega_{1} x_{4}\right)\right)=n \frac{\pi}{2}(24) \\
n=1,3,5,7, \ldots \ldots \ldots \\
k_{1} x_{1}^{\prime}-\omega_{1} x_{4}^{\prime}=n \frac{\pi}{2}-\left(k_{1} x_{1}-\omega_{1} x_{4}\right)(25) \\
\Lambda=-\frac{24 \pi G}{c} \sin \frac{n \pi}{2} \sin \left(\frac{n \pi}{2}-2\left(k_{1} x_{1}-\omega_{1} x_{4}\right)\right)
\end{gathered}
$$

Every group of nuclei have a level number $n$ and if $n=1$, we find

$$
\Lambda=-\frac{24 \pi G}{c} \sin \left(\frac{\pi}{2}-2\left(k_{1} x_{1}-\omega_{1} x_{4}\right)\right)
$$

If $Z=Z^{\prime}=Z_{0}$ then $\Lambda=0$ and

$$
\frac{\pi}{2}-2\left(k_{1} x_{1}-\omega_{1} x_{4}\right)=m \pi(28)
$$

$m=0,1,2,3, \ldots \ldots \ldots$

For every sublevel number $m$ we have a subgroup of nuclei, this subgroup is the ground state and their isobars and for $m=0$, we have

$$
\begin{gathered}
\frac{\pi}{2}-2\left(k_{1} x_{1}-\omega_{1} x_{4}\right)=0 \\
\left(k_{1} x_{1}-\omega_{1} x_{4}\right)=\frac{\pi}{4}(30)
\end{gathered}
$$

Let $\mathrm{n}=3$, we find

$$
\Lambda=\frac{24 \pi G}{c^{4}} \sin \left(\frac{3 \pi}{2}-2\left(k_{1} x_{1}-\omega_{1} x_{4}\right)\right)
$$

If $Z=Z^{\prime}=Z_{0}$ then $\Lambda=0$ and then

$$
\frac{3 \pi}{2}-2\left(k_{1} x_{1}-\omega_{1} x_{4}\right)=m \pi
$$

If $m=0$, we have

$$
\left(k_{1} x_{1}-\omega_{1} x_{4}\right)=\frac{3 \pi}{4}
$$

Cosmological constant is the curve of sine function and its interval values are given by

$$
\left(-\frac{24 \pi G}{c^{4}} \leq \Lambda \leq \frac{24 \pi G}{c^{4}}\right)
$$

\section{Conclusions}

Universe is a nuclear reactor contains a huge collection of nuclei at a wide range. These nuclei classified into groups of nuclei and each group has a collection of subgroups.

\section{References}

1. Mohammed A El-Lakany (2017) Unification of Gravity and Electromagnetism. Journal of Physical Science and Application 7(3): 15-24.

2. Ramana Murthy PV, Wolfendale AW (2010) Gamma-ray Astronomy. $2^{\text {nd }}(E d n$.$) , Cambridge University Press, USA.$

3. Schroder FG (2012) Instruments and Methods for the Radio Detection of High Energy Cosmic Rays. $1^{\text {st }}$ (Edn.), Springer Theses, Springer-Verlag Berlin Heidelberg, Germany, pp: 190.

4. Wei- Chau Xie (2010) Differential equations for engineers. Cambridge University Press, USA, pp: 45.

5. Ogio S (2004) The energy spectrum and chemical composition of primary cosmic rays with energies from $10^{14}$ to $10^{16} \mathrm{ev}$. The Astrophysical Journal 612(1): 268275.

6. Greiner W, Muller B (1994) Quantum Mechanics, Symmetries. Auflage Spriger-Verlag Berlin Heidelberg, New York USA.

7. Schneider P (2006) Extragalactic Astronomy and Cosmology. 1st (Edn.), Introduction and overview. Springer Berlin Heidelberg, New York, USA.

8. Liddle A (2013) An introduction to modern cosmology. 2nd (Edn.), Willey Online library, pp: 192.

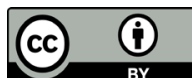

